\title{
BINARIES IN THE UNIVERSE. DYNAMICAL MECHANISMS OF FORMATION AND EVOLUTION OF DIFFERENT KINDS OF CONSTITUENTS OF THE UNIVERSE
}

\author{
J. ANOSOVA ${ }^{1}$, K.TANIKAWA ${ }^{1}$, Y.FUNATO ${ }^{2}$ \\ 1.National Astronomical Observatory, Mitaka, Tokyo 181,Japan; \\ 2.Tokyo University, Komaba Meguro, Tokyo 153, Japan
}

We study the dynamics of extended shells of relatively low-mass particles around and inside the orbit of two heavy centres of gravity (a binary) by computer simulations. The binary components are surrounded by $\mathrm{N}=16000$ small mass particles in a uniform random distribution on a few spherical envelopes with different radii expanding with some velocity. Some shells are inside the orbit of the binary.

The shell expands isotropically with decreasing velocity, and then falls back into the system forming zones of compressed matter. At some moment of time there could be a collapse of these particles on to the heavier component of the binary. Further on in time, some part of those particles which were outside the binary orbit escape from the system. Other particles which were initialy inside of the orbit are captured by binary components.

We consider a number of different models with different initial parameters. For models with smaller radii of shells, about one-half the particles escape from systems; while for larger values the shell disrupts as a whole. Escaping particles form collimated flows in the plane of orbit of the binaries. Positions of flows and directions of motion depend on positions of heavier components of binaries at the moment of a closest approach of particles and on ratios of masses of binary components.

We show that during evolution of our models different kinds of structures of systems often are very similar to the observed structures of galaxies: spiral and elliptical galaxies, interacting galaxies, different kinds of flows and jets. Totally, systems are expanding - after 40 periods of rotation of the binary the system expands by about 300 times. 